\section{Case Reports in Ophthalmology}

Case Rep Ophthalmol 2020;11:546-552

DOI: 10.1159/000509721

Published online: October 29, 2020
(C) 2020 The Author(s)

Published by S. Karger AG, Basel www.karger.com/cop

This article is licensed under the Creative Commons Attribution-NonCommercial 4.0 International License (CC BY-NC) (http://www.karger.com/Services/OpenAccessLicense). Usage and distribution for commercial purposes requires written permission.

\title{
Early Progressive Circumpapillary Lesion as Atypical Presentation of Multiple Evanescent White Dot Syndrome: A Case Report
}

\author{
José Ignacio Vela ${ }^{a-c}$ Clément Jean Arthur Marie Passabosc ${ }^{a, c}$ \\ José Antonio Buil Calvo ${ }^{a, c}$ \\ a Ophthalmology Department, Hospital de la Santa Creu i de Sant Pau, Barcelona, Spain; \\ bInstitut Comtal d'Oftalmologia, Barcelona, Spain; 'Universitat Autònoma de Barcelona \\ UAB, Barcelona, Spain
}

\section{Keywords}

Atypical presentation - Circumpapillary lesion - Giant white spot - Multiple evanescent white dot syndrome

\begin{abstract}
Classical clinical findings of multiple evanescent white dot syndrome (MEWDS) include multiple, small white dots scattered throughout the posterior pole, foveal granularity, posterior vitreous cells, and mild optic disc swelling. We describe the case of a 35-year-old man who was admitted to our department with an unusual presentation of MEWDS at the early onset of the disease. A unilateral circumpapillary retinal white spot was observed. Spectral domain optical coherence tomography demonstrated irregularities of the retinal pigment epithelium and disruptions of the outer retinal layers around the optic nerve without other abnormalities. A few days later, the lesion spread centrifugally from the peripapillary region and along the vascular arcades. This distinctive appearance in an early stage of the disease may suggest a disorder other than MEWDS, which can lead to a misdiagnosis and unnecessary treatment.




\section{Case Reports in Ophthalmology}

Vela et al.: Early Progressive Circumpapillary Lesion as Atypical Presentation of Multiple Evanescent White Dot Syndrome: A Case Report

\section{Introduction}

Multiple evanescent white dot syndrome (MEWDS) is a rare condition of unknown etiology whose clinical characteristics have been well described [1]. Its clinical spectrum has expanded in recent years, including atypical presentations [2]. Various peripapillary findings have been reported in MEWDS, including persistent indocyanine green angiography (ICGA) hypofluorescence, choroidal neovascularization, simultaneous presentation with multifocal choroiditis, and peripapillary serous detachments [3-6].

Herein, we describe an unusual presentation of MEWDS at the early onset of the disease showing a progressive circumpapillary retinal white spot that can lead to initial misdiagnosis.

\section{Case Presentation}

A healthy 35-year-old man was admitted to our emergency department with a 3-day history of enlarging temporal scotoma in his left eye. He denied any recent illness. Corrected visual acuity was 20/20 in both eyes. Results of examination and testing of the right eye were normal. The left eye showed an annular zone of peripapillary outer retinal whitening with a discrete margin (Fig. 1). There were no vitreous cells, optic disk swelling, or foveal granularity. No white dots were seen clinically. Fundus autofluorescence showed mild hyperautofluorescence, especially at the margins of the lesion (Fig. 2). Spectral domain optical coherence tomography (SD-OCT) demonstrated irregularities of the retinal pigment epithelium and disruptions of the outer retinal layers with attenuation of the external limiting membrane around the optic nerve without abnormalities in the macula (Fig. 3). Three days later, the lesion spread centrifugally from the peripapillary region and along the vascular arcades. At this time, SD-OCT showed lesions involving the macula. The patient refused to have a fluorescein angiography and ICGA. A blind spot enlargement and central scotoma was observed in the Goldmann visual field. Diagnostic considerations included MEWDS, multifocal choroiditis with panuveitis, acute posterior multifocal placoid pigment epitheliopathy (APMPPE), early-stage serpiginous choroiditis, tuberculosis (as cause of serpiginous-like choroidopathy), syphilis (as masquerader disorder), and acute zonal occult outer retinopathy (AZOOR). Results of an intensive laboratory workup were unremarkable. It included blood and chemistry examinations, angiotensin-converting enzyme, complement and rheumatoid factor levels, and serology tests for hepatotropic viruses. Treponemal test, tuberculin skin test, QuantiFERON-TB, cytomegalovirus, and human immunodeficiency virus were also ruled out. Prednisone, $60 \mathrm{mg}$ per day orally, was prescribed.

One week later, the circumpapillary white spot extended to the equator and the few satellite dots at the level of the deep retina had evolved into multiple larger white dots characteristic of MEWDS. Foveal granularity was also observed. At that moment, the patient reported having a similar episode in the right eye 9 years earlier (Fig. 4). Hypoautofluorescence of inactive lesions and thickening of the outer retina and choroid, typical of serpiginous choroiditis, were not seen. Chorioretinal scars nasally to the optic disc, characteristic of multifocal choroiditis with panuveitis, could not be observed. Prednisone was then tapered for 1 month. By 3 months, disappearance of the white dots, the circumpapillary spot, and foveal granularity was finally observed with complete resolution of symptoms. 


\section{Case Reports in Ophthalmology}

\begin{tabular}{l|l}
\hline DOI: $10.1159 / 000509721$ & (c) 2020 The Author(s). Published by S. Karger AG, Basel
\end{tabular} www.karger.com/cop

Vela et al.: Early Progressive Circumpapillary Lesion as Atypical Presentation of Multiple Evanescent White Dot Syndrome: A Case Report

\section{Discussion}

Classical clinical findings of MEWDS include multiple, small white dots scattered throughout the posterior pole, foveal granularity, posterior vitreous cells, and mild optic disc swelling [1]. The clinical spectrum of this syndrome has expanded in recent years and atypical cases have been described [2]. Overlap may be seen with other entities. In fact, it has been recommended that MEWDS should be part of the AZOOR complex [7].

Gass et al. [8] proposed that AZOOR viral introduction in the retina may occur at the optic nerve margin because of the absence of surrounding neuroepithelium at this site. The optic nerve swelling and the peripapillary findings reported in MEWDS, especially the typical zonal peripapillary hypofluorescence observed in multiple ICGA studies, suggest that this site is important in the early stages of the disease [9]. Besides, it seems to be responsible for symptoms such as loss of vision, scotoma, and photopsia.

Our case showed a confluent circumpapillary retinal white spot without optic nerve swelling or foveal granularity, which represents an unusual presentation of MEWDS at the early onset of the disease (only 3 days after the onset of symptoms). This early stage is probably missed in most cases because patients usually have mild symptoms and delay their referral to the ophthalmologist. Moreover, multimodal imaging is often scheduled a few days later. Cahuzac et al. [10] reported 4 patients seen within 1 week of onset. They suggested that ellipsoid zone disruption starts simultaneously around the optic nerve and in a very restricted submacular area before extending diffusely. They called this early stage "hyper-early" MEWDS. However, white spots were noted in the 3 cases, and foveal granularity was found in all of them. The macular region was not affected in our case (as shown in Fig. 3a), indicating that optic nerve involvement probably precedes the macula.

Similar cases describing a "giant white spot" have been reported. Ray and Loewenstein [11] reported a larger white peripapillary outer retinal lesion that evolved into a more classical MEWDS appearance of the fundus. Barile et al. [12] described a similar case with an acute transient fluorescein angiographic staining of the peripapillary chorioretinopathy, and Jampol et al. [1] suggested that these geographic lesions may represent a confluence of more typical MEWDS spots. Nonetheless, fundus autofluorescence or SD-OCT imaging were not performed in any case reported.

Case series of atypical presentations of MEWDS with peripapillary whitening/mild disk swelling without characteristic white dots have been reported [2]. Unlike our case, foveal granularity was present in all patients. Three cases presenting a progressive circumpapillary MEWDS were described in severe cases of this syndrome [13]. All of them showed typical wreath-like satellite spots around the large lesion. No satellite lesions were observed in our patient at the beginning. This fact probably indicates that our case represents an early stage of the disease.

The initial distinctive appearance of this peripapillary spot can make an accurate diagnosis more difficult. In addition, an inappropriate or unnecessary treatment can be prescribed, as occurred in our case.

In conclusion, our case demonstrates the peripapillary SD-OCT involvement as initial manifestation of MEWDS. Ophthalmologists should be aware of this early stage of MEWDS to avoid initial misdiagnosis in an otherwise benign disease. 


\section{Case Reports in Ophthalmology}

\begin{tabular}{l|l}
\hline DOI: $10.1159 / 000509721$ & (c) 2020 The Author(s). Published by S. Karger AG, Basel
\end{tabular}
www.karger.com/cop

Vela et al.: Early Progressive Circumpapillary Lesion as Atypical Presentation of Multiple Evanescent White Dot Syndrome: A Case Report

\section{Statement of Ethics}

Verbal and written informed consents were obtained from the patient for publication of this case report and all accompanying images.

\section{Conflict of Interest Statement}

The authors have no conflict of interest to declare.

\section{Funding Sources}

No funding sources or sponsors exist in this paper.

\section{Author Contributions}

J.I.V. and C.J.A.M.P. contributed to the conception and co-wrote the manuscript. J.A.B.C. provided clinical revision of the article. All authors approved the final version of the manuscript.

\section{References}

1 Jampol LM, Sieving PA, Pugh D, Fishman GA, Gilbert H. Multiple evanescent white dot syndrome. I. Clinical findings. Arch Ophthalmol. 1984 May;102(5):671-4.

2 Shelsta HN, Rao RR, Bhatt HK, Jampol LM. Atypical presentations of multiple evanescent white dot syndrome without white dots: a case series. Retina. 2011 May;31(5):973-6.

3 Yen MT, Rosenfeld PJ. Persistent indocyanine green angiographic findings in multiple evanescent white dot syndrome. Ophthalmic Surg Lasers. 2001 Mar-Apr;32(2):156-8.

4 Rouvas AA, Ladas ID, Papakostas TD, Moschos MM, Vergados I. Intravitreal ranibizumab in a patient with choroidal neovascularization secondary to multiple evanescent white dot syndrome. Eur J Ophthalmol. 2007 Nov-Dec;17(6):996-9.

5 Schaal S, Schiff WM, Kaplan HJ, Tezel TH. Simultaneous appearance of multiple evanescent white dot syndrome and multifocal choroiditis indicate a common causal relationship. Ocul Immunol Inflamm. 2009 Sep-Oct;17(5):325-7.

6 Chao DL, Marsiglia M, Ahmad B, Sridhar J, Shah GK, de Souza EC, et al. Peripapillary serous detachment in multiple evanescent white dot syndrome. Retina. 2015 Mar;35(3):521-4.

7 Gass JD. Are acute zonal occult outer retinopathy and the white spot syndromes (AZOOR complex) specific autoimmune diseases? Am J Ophthalmol. 2003 Mar;135(3):380-1.

8 Gass JD, Agarwal A, Scott IU. Acute zonal occult outer retinopathy: a long-term follow-up study. Am J Ophthalmol. 2002 Sep;134(3):329-39.

9 Marsiglia M, Gallego-Pinazo R, Cunha de Souza E, Munk MR, Yu S, Mrejen S, et al. Expanded clinical spectrum of multiple evanescent white dot syndrome with multimodal imaging. Retina. 2016 Jan;36(1):64-74.

10 Cahuzac A, Wolff B, Mathis T, Errera MH, Sahel JA, Mauget-Faÿsse M. Multimodal imaging findings in 'hyperearly' stage MEWDS. Br J Ophthalmol. 2017 Oct;101(10):1381-5.

11 Ray S, Loewenstein J. Atypical manifestation of multiple evanescent white dot syndrome with large peripapillary lesion. Arch Ophthalmol. 2003 Dec;121(12):1794-6.

12 Barile GR, Reppucci VS, Schiff WM, Wong DT. Circumpapillary chorioretinopathy in multiple evanescent white-dot syndrome. Retina. 1997;17(1):75-7.

13 Luttrull JK, Marmor MF, Nanda M. Progressive confluent circumpapillary multiple evanescent white-dot syndrome. Am J Ophthalmol. 1999 Sep;128(3):378-80. 


\section{Case Reports in Ophthalmology}
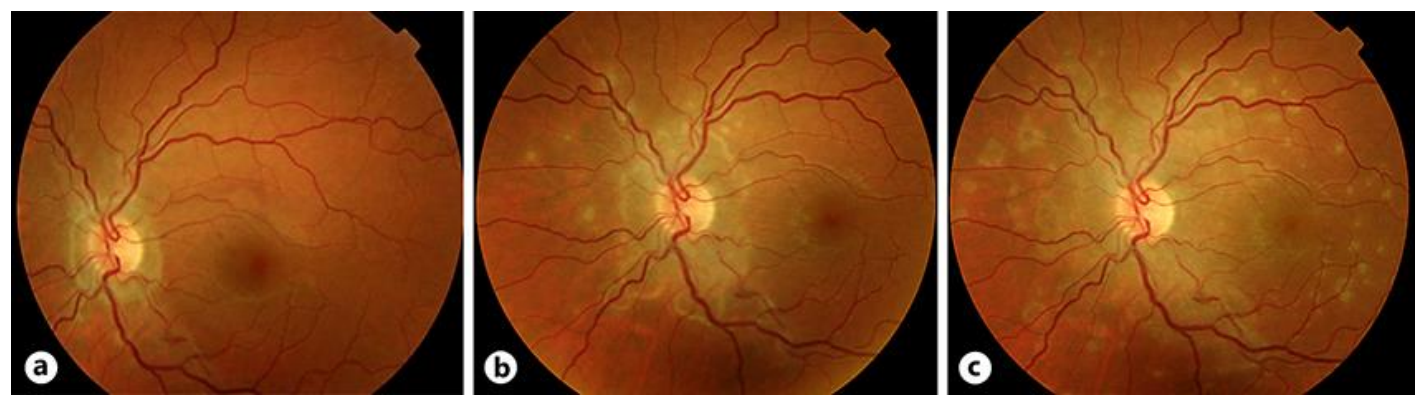

Fig. 1. a Fundus photograph of the left eye on initial examination showing a peripapillary lesion without optic disk swelling. $\mathbf{b}$ Three days later, the lesion spread centrifugally from the peripapillary region and along the vascular arcades. Note a few pinpoint satellite spots near the edge of the confluent lesion. c One week later, the circumpapillary white spot extended to the equator and evolved into characteristic lesions of MEWDS. Foveal granularity was also observed.
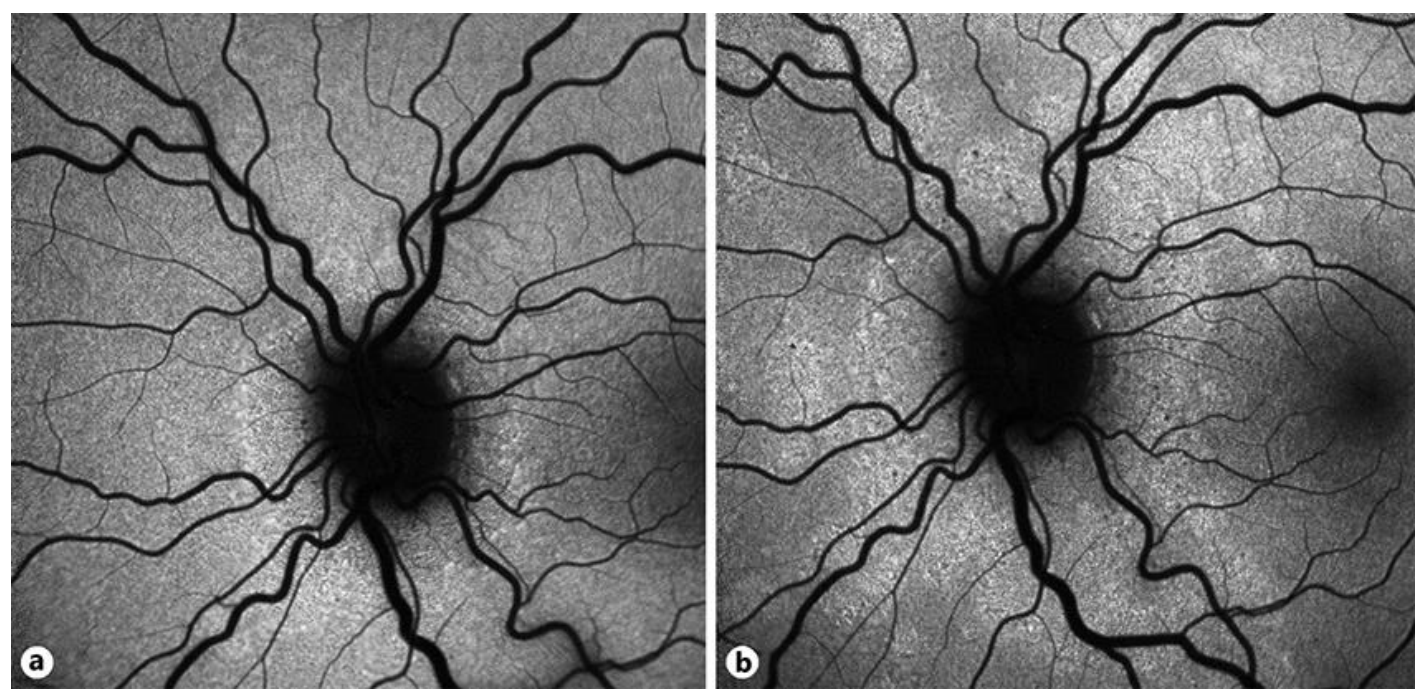

Fig. 2. a Fundus autofluorescence showing mild hyperautofluorescence in the acute phase of MEWDS. b Note the progression of the lesion after 3 days. 


\section{Case Reports in Ophthalmology}
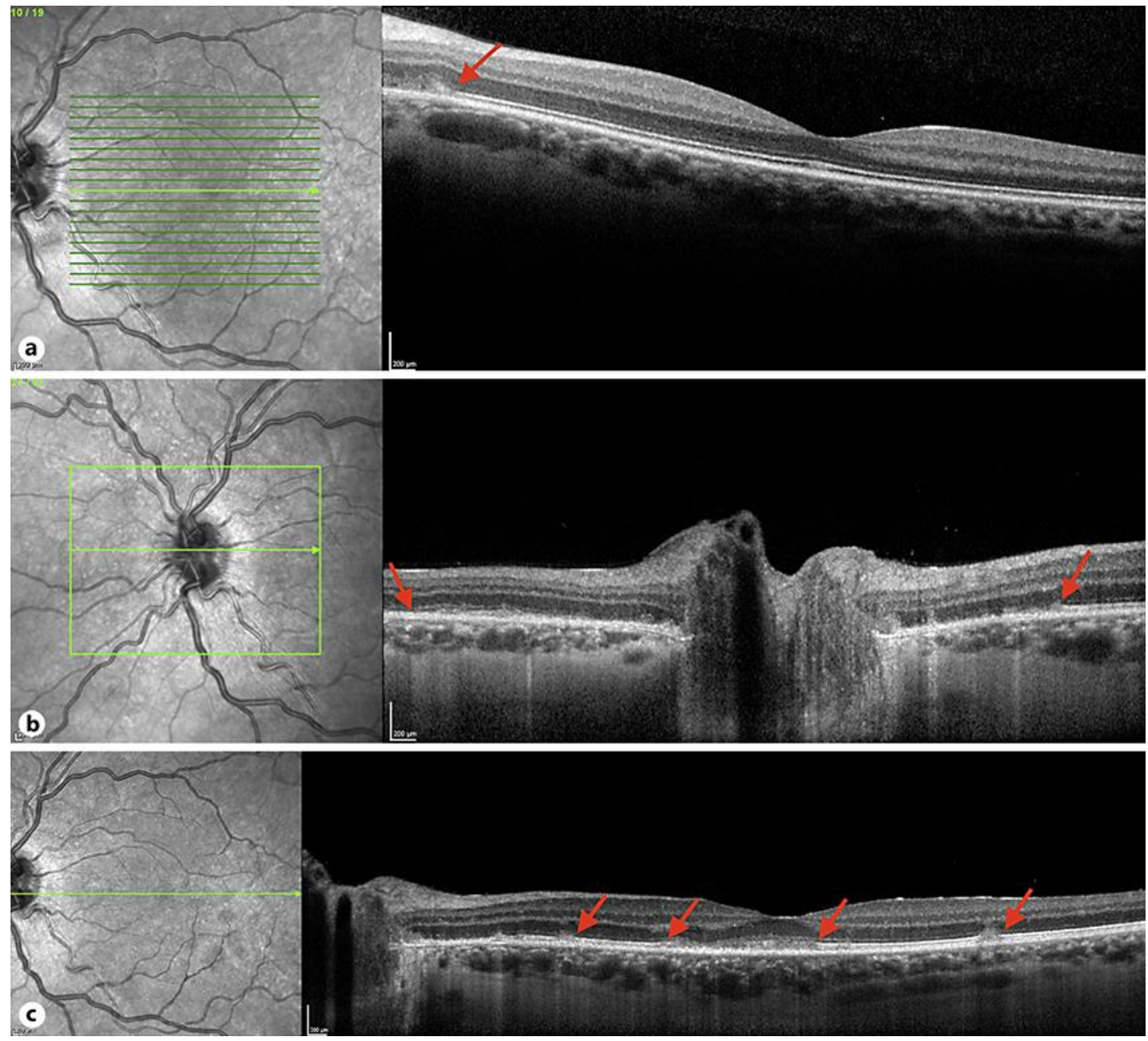

Fig. 3. a SD-OCT shows disrupted ellipsoid zone located exclusively at the peripapillary area at onset. No abnormalities in SD-OCT were seen in the macula. $\mathbf{b}$ Tomographic image focused on the optic nerve showing disruption of the ellipsoid zone between red arrows. No optic nerve swelling is observed. c One week later, SD-OCT demonstrated irregularities of the retinal pigment epithelium and disruptions of the outer retinal layers with attenuation of external limiting membrane in the subfoveal and parafoveal area (red arrows). 
Case Reports in Ophthalmology
Case Rep Ophthalmol 2020;11:546-552 DOI: 10.1159/000509721

(c) 2020 The www.karger.com/cop

Vela et al.: Early Progressive Circumpapillary Lesion as Atypical Presentation of Multiple Evanescent White Dot Syndrome: A Case Report

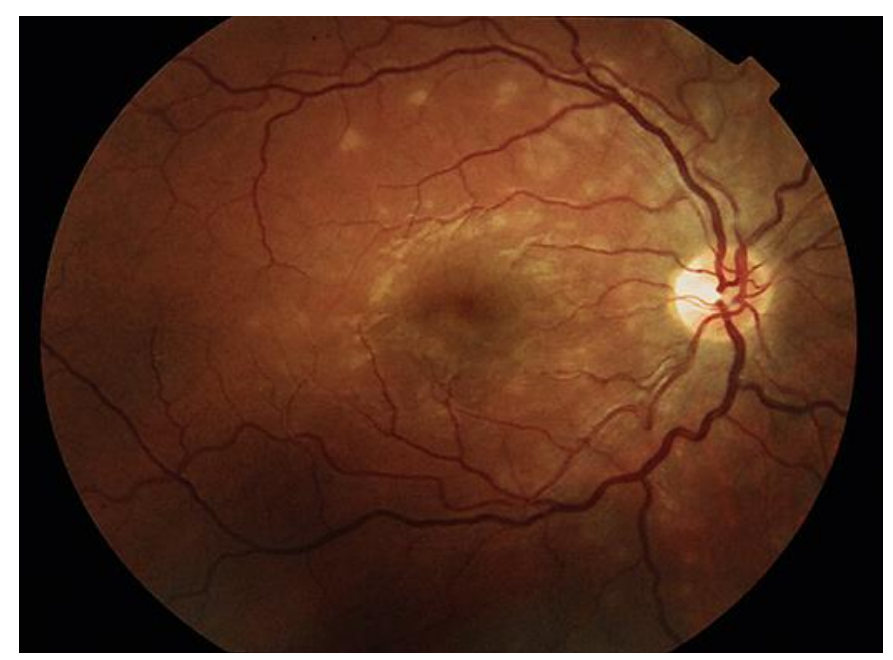

Fig. 4. Image showing typical white dots characteristic of MEWDS in the right eye 9 years earlier. 